\title{
Control of the Thermal Fractal Diffusion of Tightly Compressed Heterogeneous Layers of Thin Plates
}

\author{
Wei Qiao, ${ }^{1,2}$ Shu-Tang Liu,, and Jie Sun ${ }^{2}$ \\ ${ }^{1}$ School of Control Science and Engineering, Shandong University, Jinan 250061, China \\ ${ }^{2}$ School of Mechanical and Electrical Engineering, Shandong University at Weihai, Weihai 264209, China \\ Correspondence should be addressed to Jie Sun; sunj@sdu.edu.cn
}

Received 30 June 2014; Revised 28 September 2014; Accepted 30 September 2014; Published 5 November 2014

Academic Editor: Wang Xing-yuan

Copyright (c) 2014 Wei Qiao et al. This is an open access article distributed under the Creative Commons Attribution License, which permits unrestricted use, distribution, and reproduction in any medium, provided the original work is properly cited.

\begin{abstract}
As the thermal conductivity of thin plates composed of tightly compressed heterogeneous layers varies continuously in the form of an exponential function, we present a nonlinear dynamical model of the fractal growth of thermal diffusion. We also analyze the quantitative relationship between the probability of growth and the disturbance term, predict the control action of the environmental disturbance term on fractal growth, and use Matlab simulation to verify the control effectiveness of thermal fractal diffusion. The results facilitate the selection of appropriate control areas and control parameters for the thermal diffusion variable coefficients. In addition, variation in the fractal dimension reflects the influence of environmental disturbance on the complex process of thermal fractal diffusion.
\end{abstract}

\section{Introduction}

Fractal theory has attracted considerable research interest [18], with the diffusion-limited aggregation (DLA) [9] model proposed by Witten and Sander receiving particular attention. The DLA model successfully simulates fractal growth processes under far-from-equilibrium conditions. It has been widely used to explain various growth and aggregation phenomena relating to fractal morphologies and has met with widespread enthusiasm from researchers $[10,11]$. Shen [12] used an improved DLA model to effectively control fractal thermal diffusion using a two-dimensional constant coefficient. Zhang and Liu [13] interpreted the thermal conductivity of homogeneous material as a constant and examined methods of controlling the thermal diffusion fractal growth of thin plates under conditions of environmental disturbance.

However, the thermal conductivity of a material is not usually a constant. In most cases, including that of the widely used thin plates created from homogeneous layers, the level of conductivity changes constantly in accordance with the structural make-up, orientation, chemical components, and temperature. Contrary to the assumptions of many researchers [14-18], changes in thermal source, pressure, and many other external factors in real and complex environments affect the aggregation growth process of fractal diffusion, resulting in a fractal growth morphology that is complicated, variable, and difficult to predict. It is thus important to identify effective methods of predicting and controlling fractal growth in nonhomogeneous plates.

In this paper, we are concerned with the continuous variation of thermal conductivity in the form of an exponential function and with thermal fractal diffusion in the tightly compressed heterogeneous layers of thin plates. We also analyze the quantitative relationship between the growth probability and the disturbance term. Constructing an environmental disturbance term that comprises a forced item with a polynomial form and a source item with a circular designated area enables us to predict and control the thermal fractal diffusion of thin plates made of homogeneous layers.

\section{Thermal Diffusion System of the Nonhomogeneous Layers of Thin Plates}

In $W$ (see Figure 1), a thin plate comprised of tightly compressed heterogeneous layers, each layer contains various substances, all with different levels of thermal conductivity. The layers are tightly compressed to remove transition 


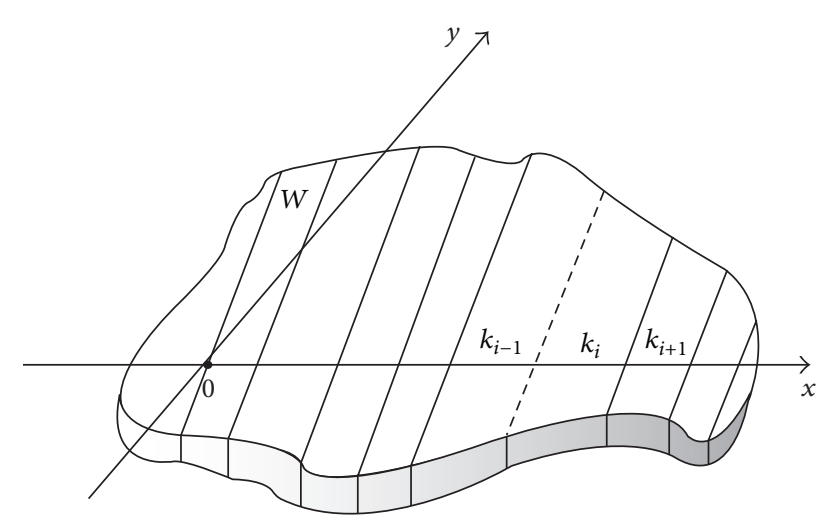

FIgURE 1: Thermal conductivity $k_{i}$ of a thin plate of tightly compressed heterogeneous layers.

resistance. The plate's thermal-conduction equation is as follows: $\rho c(\partial V / \partial t)=(\partial V / \partial x)(a(x, y)(\partial V / \partial x))+(\partial V / \partial y)(b(x$, $y)(\partial V / \partial y))$, where $\rho$ refers to the density of the substance, $c$ refers to the specific heat, and $a(x, y)$ and $b(x, y)$ refer to thermal conductivity [19].

We arrange the tightly compressed heterogeneous layers of the thin plate in spatial order to enable the use of a step approximation for the plate's varying thermal conductivity. As shown in Figure 2, the solid line represents the thermal conductivity, $k_{i}$, and the dashed line represents the approximately continuous function $K(x)$. We assume that the thermal conductivity, $x$, varies and meets the following conditions: $a(x, y)=b(x, y)=k_{0} e^{\alpha x}, k_{0}>0$, where $\alpha$ is a constant to be identified. When $\alpha>0, x$ steps up; when $\alpha<0$, $x$ steps down; when $\alpha=0$, the thin plate of nonhomogeneous layers is composed of the same material $[20,21]$.

2.1. Mathematical Model. If the tightly compressed heterogeneous layers of thin plates contain thermal sources, the dynamics of fractal growth allow us to represent the probability of growth in the thermal diffusion process as a distributed-parameter system conforming to the following boundary conditions:

$$
\begin{aligned}
& \frac{\partial V}{\partial x}\left(a(x, y) \frac{\partial V}{\partial x}\right)+\frac{\partial V}{\partial y}\left(b(x, y) \frac{\partial V}{\partial y}\right) \\
& \quad=u(x, y)+f(V(x, y)),
\end{aligned}
$$

where $V(x, y)$ represents the probability of thermal diffusion (temperature) [22] at a certain time, when the spatial body $W$ is located at $(x, y)$. The function $f(\cdot)$ is usually a nonlinear function known as the "forced" term, and $u$ is a quantitative initial condition called the "source" term. Both the nonlinear disturbance term $f(\cdot)$ and the source term $u$ can be considered equivalent to the environmental disturbance term $G$.

Given the complexity of (1), which depicts thermal diffusion with variable coefficients, we cannot analytically determine the solution. However, we can use the finite difference scheme to calculate a numerical solution for the thermal diffusion equation. In accordance with the finite volume

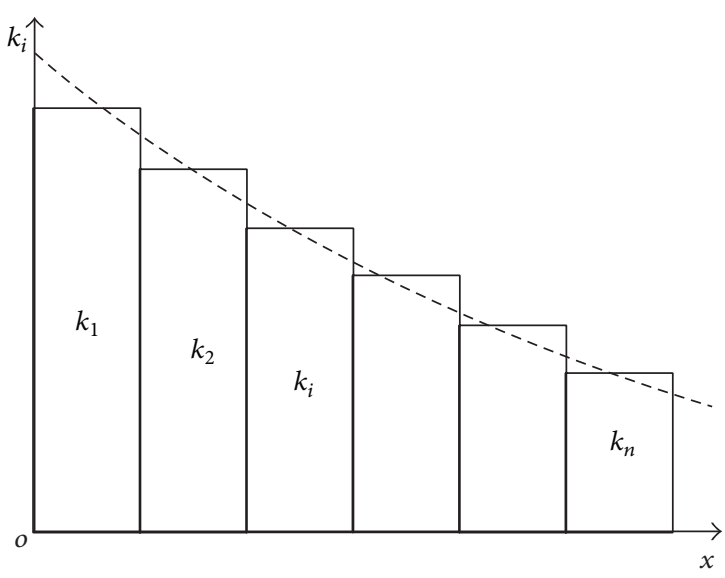

FIGURE 2: Spatial arrangement of the varying thermal conductivity of a thin plate comprising tightly compressed heterogeneous layers.

method [23], the discrete dynamic structure of System (1), a nonlinear fractal growth system, can be represented as follows:

$$
\begin{aligned}
& a_{i+(1 / 2), j} V_{i+1, j}+a_{i-(1 / 2), j} V_{i-1, j}+b_{i, j-(1 / 2)} V_{i, j-1}+b_{i, j+(1 / 2)} V_{i, j+1} \\
& \quad-\left(a_{i+(1 / 2), j}+a_{i-(1 / 2), j}+b_{i, j-(1 / 2)}+b_{i, j+(1 / 2)}\right) V_{i, j} \\
& =u_{i, j}+f\left(V_{i, j}\right) .
\end{aligned}
$$

Without loss of generality, we assume that $u(x, y)$ and $f(x, y)$ are smooth functions and ensure that the values of $u(x, y)$ and $f(x, y)$ within the spatial area of thermal diffusion are sufficiently small. To ensure that adding the source term and the forced term does not negate the compliance of the difference equation with the extremum principle, $V(x, y)$ can only obtain an extremum at the boundary, which requires it to meet the following boundary conditions. First, the far-from-internal $V(x, y)$ must be capable of providing the surface with a sustained total particle flow, $V(x, y) \equiv 1$. Second, the arrival probability of the internal $V(x, y)$ at the boundary point where the particles terminate their growth must be $V(x, y)=0$. As $u(x, y)$ and $f(x, y)$ are sufficiently small, $0 \leq a_{i+r-(1 / 2), j} V_{i+r, j}+a_{i-r+(1 / 2), j} V_{i-r, j}+b_{i, j-r+(1 / 2)} V_{i, j-r}+$ $b_{i, j+r-(1 / 2)} V_{i, j+r}<1$ holds in the aggregation region.

2.2. Thermal Diffusion Simulation. The thermal diffusion of a thin plate consisting of tightly compressed heterogeneous layers is a random process controlled by a temperature gradient. The specific algorithm for thermal diffusion fractal growth is obtained as follows.

(1) Set the initial conditions. Establish the aggregation region $L \times L$ for the simulated thermal particles, the particlemovement step size, and the number of aggregation particles.

(2) Calculate the temperature gradient. In accordance with System (2), a discrete system, and combining the central particles subject to the initial boundary conditions $(V=0)$ with the surrounding points at infinity $(V=1)$, we can 
calculate the distribution of the temperature gradient $\nabla V$ across the whole thin plate after multiple iterations [24].

(3) Calculate the thermal-growth probability of the points awaiting growth. The growth-probability function of every possible aggregation point has a more nonlinear than linear relationship with the temperature gradient. Pietronero and Wiesmann proposed the following model of growth probability [25]:

$$
p_{i}=\frac{\left|\nabla V_{i}(x, y)\right|}{\sum_{i=1}^{n}\left|\nabla V_{i}(x, y)\right|}
$$

where $n$ refers to the number of points awaiting growth and the denominator refers to the total probability and normalization coefficient of the particles growing in all directions.

(4) Choose the particle aggregation location. Around the nucleation particles, there are both nearest neighbor and next-nearest neighbor points awaiting growth (as shown in Figure 3). During the practical process of conduction, the next-nearest neighbor is also of great importance.

The final aggregation position of the thermal particles occurs at random, and its probability is proportional to the probability $p_{i}$ of the nodes awaiting growth. We use the roulette wheel selection algorithm [26] to choose new thermal diffusion points from all of the possible points awaiting growth. $\alpha$ is a randomly produced number with a value interval of $(0,1)$. The comparison is as follows: $\sum_{i=1}^{k-1} p_{i} \leq$ $\alpha<\sum_{i=1}^{k} p_{i}$.

(5) Modify the boundary conditions. When new particles aggregate, the thermal-diffusion probability falls to zero. The thermal-diffusion probability of these newly developed points is also included among the boundary conditions.

By modifying the movement-boundary conditions of the mathematical model and using the roulette wheel selection algorithm, the model described in this paper more accurately simulates the thermal diffusion of thin plates consisting of tightly compressed heterogeneous layers.

\section{Fractal Diffusion Control of the Thermal Sources of Thin Plates Consisting of Homogeneous Layers}

The polynomial function approximation is widely used in the fields of fractal graphics, voice-processing systems, geotechnical engineering, and electrical engineering and in real physical systems such as ion-exchange glass, ion chemistry, and thin-film preparation [27].

According to the Weierstrass theorem, any continuous function defined in a closed interval can be uniformly approximated by a polynomial function. Therefore, we assume that System (2) takes the nonlinear function $f\left(V_{i, j}\right)=\xi\left(\omega V_{i, j}\right)^{p}+\mu$, and here $p=1,2, \ldots, n$. We can thus represent the system as follows:

$$
\begin{aligned}
& a_{i+(1 / 2), j} V_{i+1, j}+a_{i-(1 / 2), j} V_{i-1, j}+b_{i, j-(1 / 2)} V_{i, j-1} \\
& \quad+b_{i, j+(1 / 2)} V_{i, j+1}-\omega V_{i, j}=u_{i, j}+\mu+\xi\left(\omega V_{i, j}\right)^{p}+\omega V_{i, j} .
\end{aligned}
$$

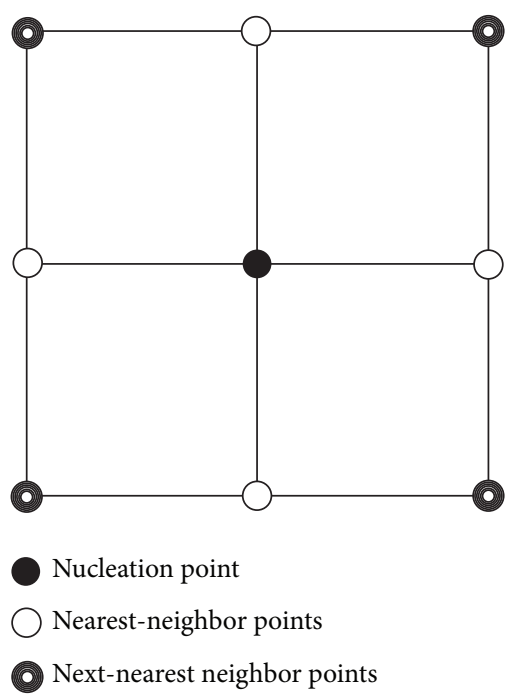

Figure 3: Nearest neighbor and next-nearest neighbor points awaiting growth.

For simplicity of presentation, the constant $\mu=0$ is assumed and the following notation is used:

$$
\begin{aligned}
E(r)= & E_{i, j}(r)=a_{i+r-(1 / 2), j} V_{i+r, j}+a_{i-r+(1 / 2), j} V_{i-r, j} \\
& +b_{i, j-r+(1 / 2)} V_{i, j-r}+b_{i, j+r-(1 / 2)} V_{i, j+r} .
\end{aligned}
$$

Consequently, System (2) takes the following form: $E(1)=u_{i, j}+\xi E^{p}(0)+E(0), p=1,2, \ldots, n . p \in Z^{+}$.

Without loss of generality, we represent the system as follows:

$$
E(r)=u(x, y)+\xi E^{p}(r-1)+E(r-1), \quad r=1,2, \ldots, n .
$$

After a simple iteration of System (6), we obtain the following:

$$
E(r)
$$

$$
\begin{array}{r}
=r u_{i, j}+\xi E^{p}(r-1)+\xi E^{p}(r-2)+\cdots+\xi E^{p}(0)+E(0), \\
=\xi\left(E^{p}(r-1)+E^{p}(r-2)+\cdots+E^{p}(0)\right)+E(0)+r u_{i, j}, \\
r=1,2, \ldots, n .
\end{array}
$$

With regard to System (7), we discuss the control action of environmental disturbance on thermal diffusion fractal growth in thin plates composed of tightly compressed heterogeneous layers. With regard to System (2), we expand $f(t)=$ $\xi t^{p}$ into a Fourier series within the interval $[-1,1]$ as follows:

$$
f(t)=\frac{a_{0}}{2}+\sum_{n=1}^{n=\infty}\left(a_{n} \cos n \pi t+b_{n} \sin n \pi t\right), \quad n=1,2, \ldots
$$

In the following discussion, we compare the effects of an odd $p$ value with those of an even $p$ value. 
3.1. Control Action of Environmental Disturbance on Thermal Fractal Diffusion When $p$ Is Even. Consider

$$
\begin{gathered}
a_{0}=\frac{1}{p+1}, \\
a_{n}=\int_{-1}^{1} f(t) \cos n \pi t d t \\
=\sum_{n=1}^{\infty}\left[-2 \cos n \pi\left(\frac{p}{n^{2} \pi^{2}}+\cdots+\frac{p !}{n^{p} \pi^{p}}\right)\right], \\
f(t)=\frac{\xi}{p+1}+\xi \quad b_{n}, \\
\times \sum_{n=1}^{\infty}\left[-2 \cos n \pi\left(\frac{p}{n^{2} \pi^{2}}+\cdots+\frac{p^{p}}{n^{p} \pi^{p}}\right) \cos n \pi t\right], \\
n=1,2, \ldots
\end{gathered}
$$

Therefore, System (6) takes the following form:

$$
\begin{aligned}
E(r)= & \frac{\xi}{p+1}+\xi \\
& \times \sum_{n=1}^{\infty}\left[-2 \cos n \pi\left(\frac{p}{n^{2} \pi^{2}}+\cdots+\frac{p !}{n^{p} \pi^{p}}\right)\right. \\
& \times \cos (n \pi E(r-1))]+E(r-1)+u, \\
E(r)= & \frac{\xi}{p+1}+\xi \\
& \times \sum_{n=1}^{\infty}\left[-2 \cos n \pi\left(\frac{p}{n^{2} \pi^{2}}+\cdots+\frac{p !}{n^{p} \pi^{p}}\right)\right. \\
& \times \cos n \pi E(r-1)]+E(r-1)+u \\
\leq & \frac{r \xi}{p+1}+r \xi \sum_{n=1}^{\infty}\left[-2 \cos n \pi\left(\frac{p}{n^{2} \pi^{2}}+\cdots+\frac{p !}{n^{p} \pi^{p}}\right)\right] \\
& +E(0)+r u .
\end{aligned}
$$

$\sum_{n=1}^{\infty}\left[-2 \cos n \pi\left(p / n^{2} \pi^{2}+\cdots+p ! / n^{p} \pi^{p}\right)\right]$ appears to be an alternating series. According to Leibnitz's theorem [28], this type of series is convergent and meets the following conditions: $\sum_{n=1}^{\infty}\left[-2 \cos n \pi\left(p / n^{2} \pi^{2}+\cdots+p ! / n^{p} \pi^{p}\right)\right]=g$, $\left(p / \pi^{2}>g>0\right)$.

Therefore,

$$
\begin{aligned}
E(r) & \leq \frac{r \xi}{p+1}+r \xi g+E(0)+r u \\
& =r \xi\left(\frac{1}{p+1}+g\right)+E(0)+r u .
\end{aligned}
$$

Let $G(\xi, u, r)=r \xi(1 /(p+1)+g)+E(0)+r u$, where $u(x, y), \xi$ is the linear function of $G(\xi, u, r)$,

$$
\begin{aligned}
& G(\xi, u, r)=r \xi\left(\frac{1}{p+1}+g\right)+E(0)+r u, \\
& \frac{\partial G}{\partial \xi}=r\left(\frac{1}{p+1}+g\right)>0, \quad \frac{\partial G}{\partial u}=r>0 .
\end{aligned}
$$

According to the "judgment" method of increasing and decreasing functions in mathematical analysis, $E(r)$ increases monotonically with respect to $V_{i, j} \xi$ and $u(x, y)$, respectively.

Conclusion 1. $V_{i, j}$ increases with source item $u_{i, j}$, which means that the introduction of source item $u(x, y)$ significantly increases the particle concentration in the control directions. Thus, the particles have obvious growth advantages in the control directions.

Conclusion 2. $V_{i, j}$ increases with source item $\xi$, which means that the introduction of the nonlinear forcing term $\xi\left(\omega V_{i, j}\right)^{p}$ intensifies particle aggregation in the whole region.

As $V(x, y)$ can only reach its extrema at the boundaries, extremum conditions can only be met by modifying control parameter $\xi$ and source item $u(x, y)$ to make $V(x, y) \ll 1$. This also ensures that the system's controlled quantity is much less than its input quantity:

$$
E(r)<r \xi\left(\frac{1}{p+1}+g\right)+E(0)+r u<1 .
$$

From Conclusions 1 and 2 and Formula (13), the quantitative relations between source item $u(x, y)$ and the nonlinear forced term can be identified. When the action region of the source item is circular, Corollary 1 holds.

Corollary 1. When the action region of a source item is circular, the center of the control region is the center of the circle, the radius is $R$, and the control function is as follows: $\left.u(x, y)\right|_{x^{2}+y^{2} \leq R^{2}}=\lambda\left(x^{2}+y^{2}\right)$. When $\lambda>(1-E(0)) / r(1 /(p+$ $1)+g+1) R^{2}, \xi<u_{\max }$; that is, the particles gain an obvious control advantage in the control region.

Proof. When the number of iterations is $r$, the source item is $u(x, y)=\lambda\left(x^{2}+y^{2}\right)$, where $x^{2}+y^{2} \leq R^{2}$; then $u_{\max }=$ $\lambda R^{2}>\left((1-E(0)) / r(1 /(p+1)+g+1) R^{2}\right) R^{2}=(1-E(0)) /$ $r(1 /(p+1)+g+1)$.

It follows from Formula (13) that

$$
E(r) \leq r \xi\left(\frac{1}{p+1}+g\right)+E(0)+r u<1
$$

that is,

$$
\begin{aligned}
& r \xi\left(\frac{1}{p+1}+g\right) \\
& \quad+E(0)+r u \leq r \xi\left(\frac{1}{p+1}+g\right)+E(0)+r u_{\max }<1 .
\end{aligned}
$$


Then

$$
\xi<\frac{1-E(0)-r u_{\max }}{r(1 /(p+1)+g)},
$$

because

$$
\begin{gathered}
u_{\max }>\frac{1-E(0)}{r(1 /(p+1)+g+1)}, \\
-r u_{\max }<-\frac{1-E(0)}{1 /(p+1)+g+1} .
\end{gathered}
$$

It follows that

$$
\begin{aligned}
\xi< & \frac{1-E(0)-r u_{\max }}{r(1 /(p+1)+g)}, \\
< & \frac{1-E(0)}{r(1 /(p+1)+g)} \\
& -\frac{1-E(0)}{r(1 /(p+1)+g+1)(1 /(p+1)+g)}, \\
= & \frac{1-E(0)}{r(1 /(p+1)+g+1)}=u_{\max } .
\end{aligned}
$$

Therefore, when $u_{\max }(x, y)>\xi$, the increase in particle concentration in the control directions clearly exceeds that of the nonlinear item in the whole region. The particles thus have obvious growth advantages in the control directions.

\subsection{Control Action of Environmental Disturbance on Thermal} Fractal Diffusion When $p$ Is Odd. Consider

$$
\begin{aligned}
& a_{n}=0, \\
& b_{n}=\int_{-1}^{1} f(t) \sin n \pi t d t \\
& =\sum_{n=1}^{\infty}\left[-2 \cos n \pi\left(\frac{p}{n \pi}+\frac{p(p-1)}{n^{2} \pi^{2}}+\cdots+\frac{p !}{n^{p} \pi^{p}}\right)\right], \\
& f(t)=\xi \\
& \quad \times \sum_{n=1}^{\infty}\left[-2 \cos n \pi\left(\frac{p}{n^{2} \pi^{2}}+\cdots+\frac{p !}{n^{p} \pi^{p}}\right) \sin n \pi t\right], \\
& n=1,2, \ldots .
\end{aligned}
$$

System (6) takes the following form:

$$
\begin{aligned}
E(r)=\xi & \\
\times \sum_{n=1}^{\infty}[ & -2 \cos n \pi\left(\frac{p}{n^{2} \pi^{2}}+\cdots+\frac{p !}{n^{p} \pi^{p}}\right) \\
& \quad \times \sin n \pi E(r-1)]+E(r-1)+u,
\end{aligned}
$$

and as a result,

$$
\begin{aligned}
& E(r) \\
& =\xi \sum_{n=1}^{\infty}\left[-2 \cos n \pi\left(\frac{p}{n^{2} \pi^{2}}+\cdots+\frac{p !}{n^{p} \pi^{p}}\right) \sin n \pi E(r-1)\right]
\end{aligned}
$$

$$
\begin{aligned}
& +E(r-1)+u \\
\leq & r \xi \sum_{n=1}^{\infty}\left[-2 \cos n \pi\left(\frac{p}{n^{2} \pi^{2}}+\cdots+\frac{p !}{n^{p} \pi^{p}}\right)\right]+E(0)+r u .
\end{aligned}
$$

According to Dirichlet's theorem [28], $\sum_{n=1}^{\infty}[-2 \cos n \pi(p)$ $\left.\left.n^{2} \pi^{2}+\cdots+p ! / n^{p} \pi^{p}\right)\right]=g$ belongs to a converging series. Then, $E(r) \leq r \xi g+E(0)+r u$.

We can then state the following: $G^{\prime}(\xi, u, r)=r \xi g+E(0)+$ ru. $G^{\prime}(\xi, u, r)$ is clearly a monotonically increasing function of the variables $\xi$ and $u$.

It is easy to show that Conclusions 1 and 2 hold when the nonlinear forced term is an odd function. Proceeding according to the proof for Corollary 1 gives Corollary 2.

Corollary 2. When the action region of the source item is circular, the center of the control region is the center of the circle, the radius is $R$, and the control function is as follows: $\left.u(x, y)\right|_{x^{2}+y^{2} \leq R^{2}}=\lambda\left(x^{2}+y^{2}\right)$. When $\lambda>(1-E(0)) / r(1+$ g) $R^{2}, \xi<u_{\max }$; that is, the particles gain an obvious control advantage in the control region.

In short, a similar conclusion is reached whether $p$ is even or odd, but the condition parameters differ.

\section{Numerical Analysis of the Thermal Fractal Diffusion of Thin Plates Consisting of Homogeneous Layers}

We simulate the fractal diffusion and aggregation of 10000 thermal particles by constructing a $101 \times 101$ grid on the thin plate $(W)$ of heterogeneous layers, with the thermal source in the center of the grid $(51,51)$. In addition, we set various values for $u(x, y)$ and the radius of the action region of the source item to simulate the control action on thermal diffusion fractal growth under conditions of environmental disturbance. Figure 4 shows the morphology of thermal diffusion aggregates on the thin plate when $f=0$ and $u=0$.

4.1. Control of Thermal Diffusion under Conditions of Environmental Disturbance. According to Conclusion 1 and Corollary 1 , the particles have an obvious growth advantage in the control region when $p=2$. When the control region is enlarged, the corresponding $V_{i, j}$ also grows and the particles gain a distinct growth advantage in the circular region. Figure 5 shows that when $p=2, a(x, y)=b(x, y)=e^{0.2 x}$, $u_{\max }(x, y)>\xi$, and the thermal fractal form increases with the action region of the source item. The figure clearly illustrates that when $u_{\max }(x, y)>\xi$, the growing particles become more closely aggregated as the radius of the domain of the source item increases. When the control region of the source item is a fixed constant, we can construct fractalaggregation graphs for various control radiuses, as shown in Figure 6. According to the graph for the control region in Figure 6, particles aggregate more closely than the particles 


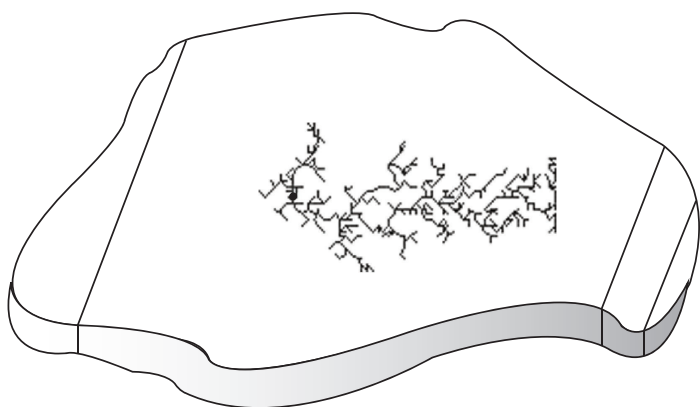

(a) $a(x, y)=b(x, y)=e^{0.2 x}$

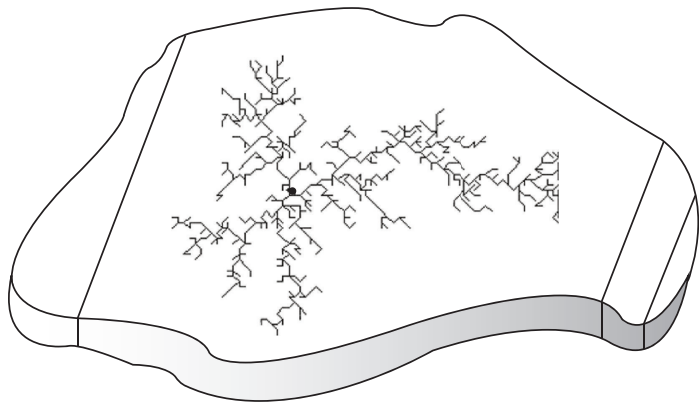

(c) $a(x, y)=b(x, y)=e^{0.02 x}$

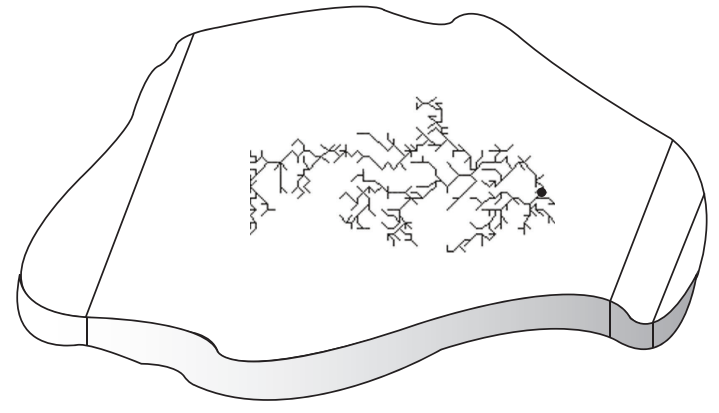

(b) $a(x, y)=b(x, y)=e^{-0.2 x}$

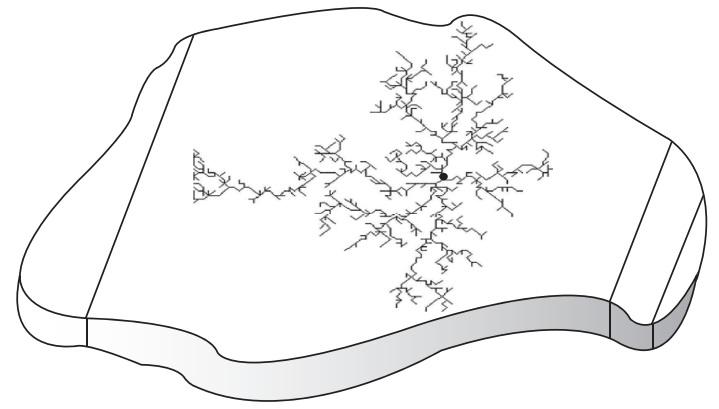

(d) $a(x, y)=b(x, y)=e^{-0.02 x}$

FIGURE 4: Thermal fractal diffusion on plate $W$ when $f=0$ and $u=0$.

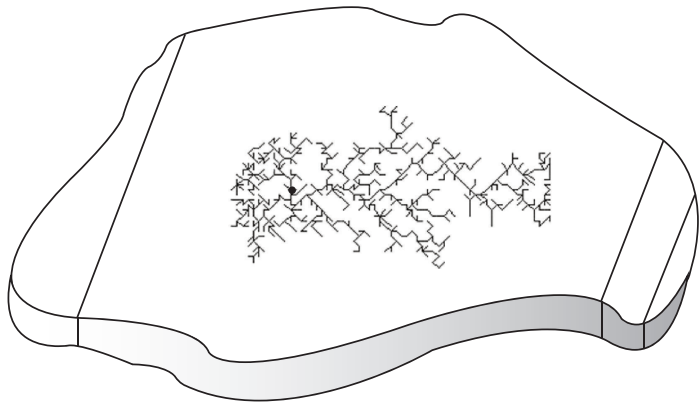

(a) $R=10$

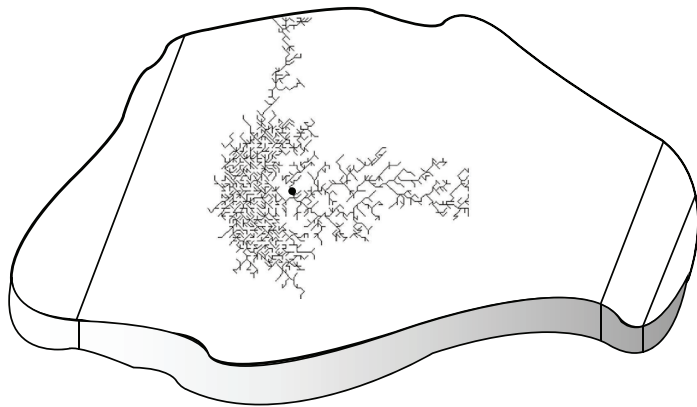

(c) $R=20$

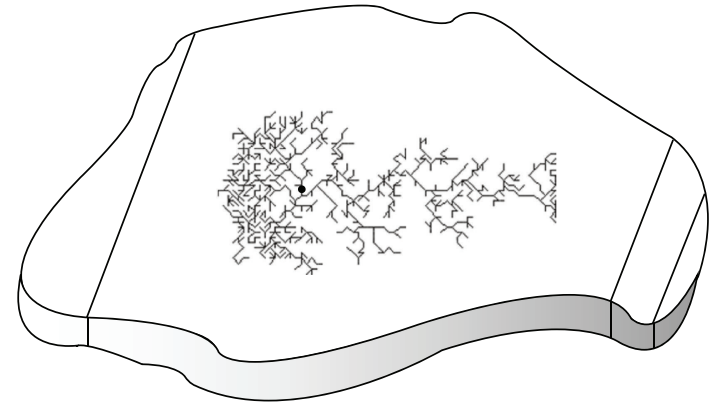

(b) $R=14$

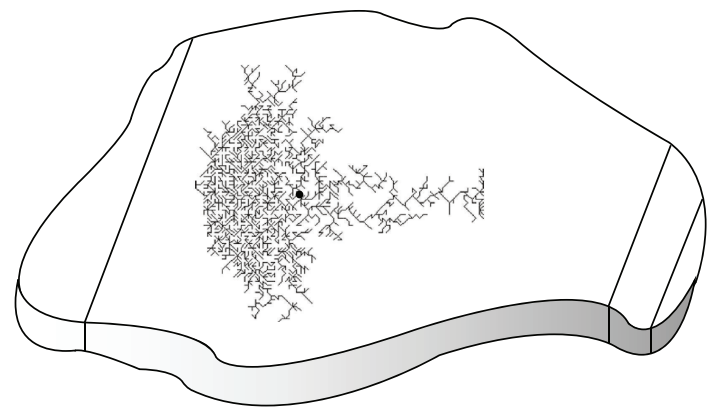

(d) $R=26$

Figure 5: Fractal aggregation when $u=5 \times 10^{-5}\left(x^{2}+y^{2}\right), \xi=1 \times 10^{-7}$, and $p=2$.

in the control region in Figure 5. This result indicates that constant control strength has a greater control action than uneven control strength.

In Figures 5 and 6, thermal conductivity to the right of the central point increases with $x$ more obviously than to the left of the central point. The thermal particles on the right grow rapidly in the direction of $x$, such that thermal conductivity in the right control region exceeds that in the left. Compared with Figure 4(a), which depicts particle aggregation in the absence of environmental disturbance, the disturbance term 


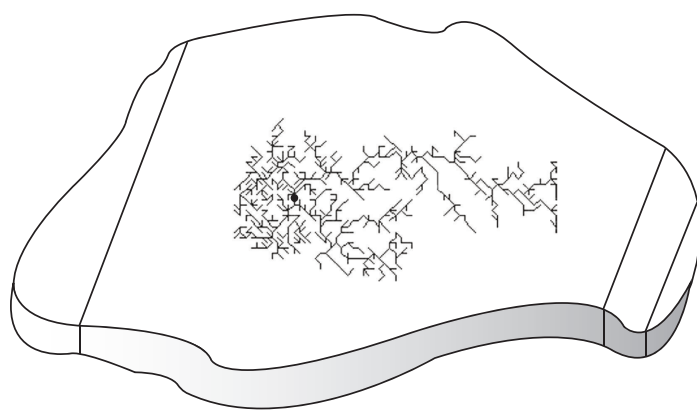

(a) $u=5 \times 10^{-3}, R=10$, and $\xi=1 \times 10^{-7}$

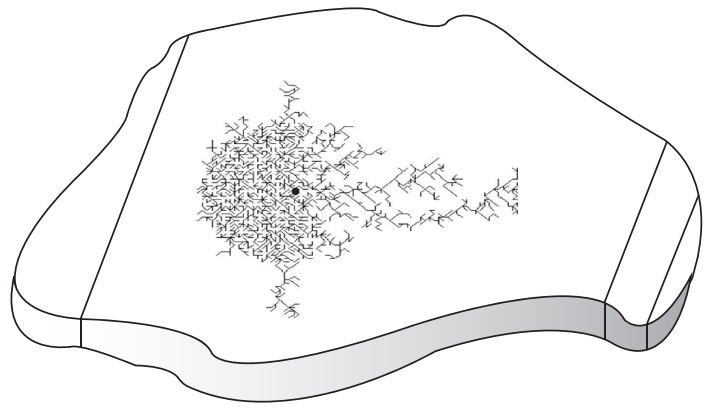

(c) $u=2 \times 10^{-2}, R=20$, and $\xi=1 \times 10^{-6}$

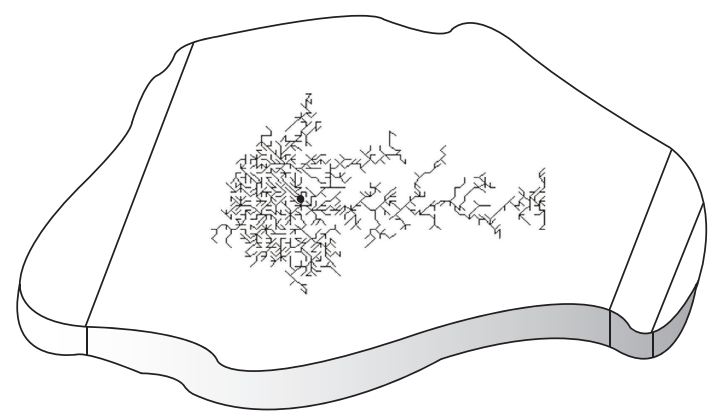

(b) $u=10^{-2}, R=14$, and $\xi=1 \times 10^{-6}$

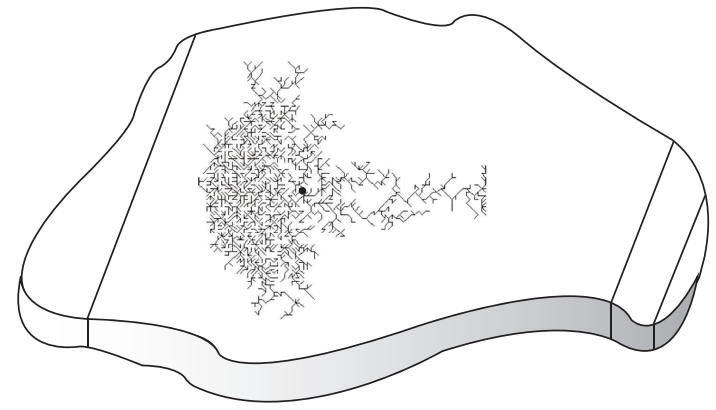

(d) $u=5 \times 10^{-3}, R=26$, and $\xi=1 \times 10^{-7}$

FigURE 6: Fractal aggregation when $u$ is a constant and $p=2$.

in Figures 5 and 6 aggregates thermal particles much more obviously in the action region.

According to Conclusion 1 and Corollary 2, particles have an obvious growth advantage in the control region when $p=3$. When the control region is enlarged, the corresponding $V_{i, j}$ also grows and the particles gain a distinct growth advantage in the circular region. Figure 7 shows that when $p=3, a(x, y)=b(x, y)=e^{0.02 x}, u_{\max }(x, y)>\xi$, and the thermal fractal form increases with the action region of the source item. Figure 8 presents the particle aggregation in various control regions when the control strength is a constant. The graph for the control region in Figure 8 shows closer aggregation in this region than that in Figure 7. Compared with Figure 4(c), which presents the thermal particle aggregation in the absence of environmental disturbance, the thermal particles in the control regions in Figures 7 and 8 are more clearly aggregated. As a result, our conclusions are similar to those for the even term. However, as thermal conductivity does not increase very clearly with $x$, the aggregation of the particles to the right of the central point in the direction of $x$ is less obvious than the equivalent aggregation in Figures 5 and 6.

4.2. Fractal Dimension of Thermal Diffusion under Conditions of Environmental Disturbance. A fractal dimension is an index for characterizing fractal patterns or sets by quantifying their complexity as a ratio of the change in detail to the change in scale. It has also been characterized as a measure of the space-filling capacity of a pattern that tells how a fractal scales differently from the space it is embedded in [29]. As the thermal diffusion of a spatial point source has an obvious
TABLE 1: $a(x, y)=b(x, y)=e^{0.2 x}$, fractal dimension of thermal diffusion under different environmental disturbances.

\begin{tabular}{lcccc}
\hline Figure number & $\xi$ & $u$ & $R$ & Fractal dimension \\
\hline Figure 4(a) & 0 & 0 & 0 & 1.2102 \\
Figure 5(a) & $10^{-7}$ & $5 * 10^{-5}\left(x^{2}+y^{2}\right)$ & 10 & 1.3557 \\
Figure 5(b) & $10^{-7}$ & $5 * 10^{-5}\left(x^{2}+y^{2}\right)$ & 14 & 1.4763 \\
Figure 5(c) & $10^{-7}$ & $5 * 10^{-5}\left(x^{2}+y^{2}\right)$ & 20 & 1.7419 \\
Figure 5(d) & $10^{-7}$ & $5 * 10^{-5}\left(x^{2}+y^{2}\right)$ & 26 & 1.8522 \\
Figure 6(a) & $10^{-7}$ & $5 * 10^{-3}$ & 10 & 1.2746 \\
Figure 6(b) & $10^{-6}$ & 0.01 & 14 & 1.3847 \\
Figure 6(c) & $10^{-6}$ & $5 * 10^{-4}$ & 20 & 1.4834 \\
Figure 6(d) & $10^{-7}$ & $5 * 10^{-3}$ & 26 & 1.7437 \\
\hline
\end{tabular}

central growth point, we use Sandbox to measure the fractal dimension with different variable coefficients and circular regions, as shown in Tables 1 and 2 . With different variable coefficient functions, and as the environmental disturbance term increases in scope and intensity, the corresponding fractal dimensions expand to different degrees. This suggests that the environmental disturbance term not only controls the surface morphological changes that occur during thermal fractal diffusion in flat objects, but also increases the complexity of fractal diffusion growth.

\section{Conclusion}

As the thermally conductive effects of thin plates consisting of homogeneous layers have extensive applications in the field 


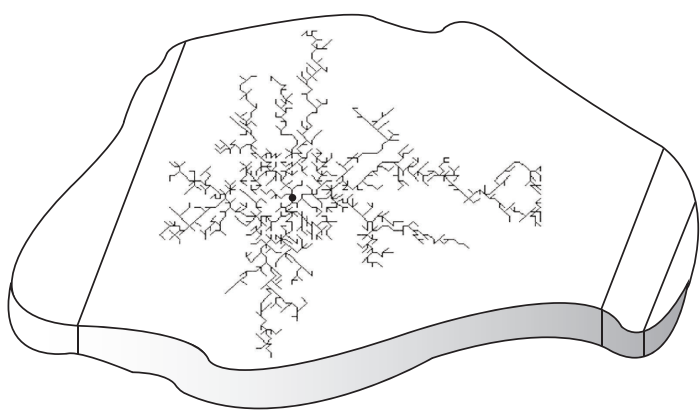

(a) $R=10$

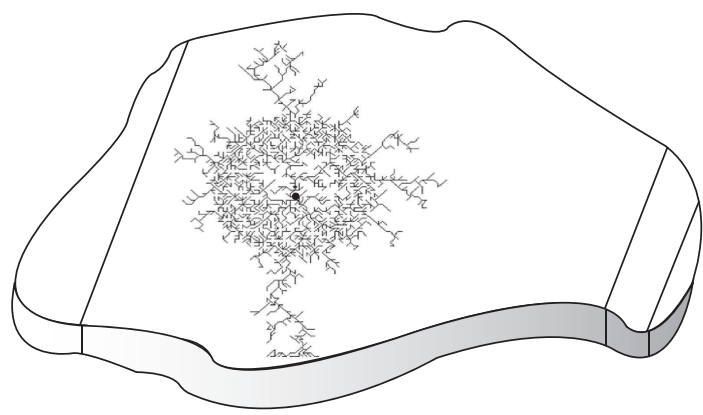

(c) $R=20$

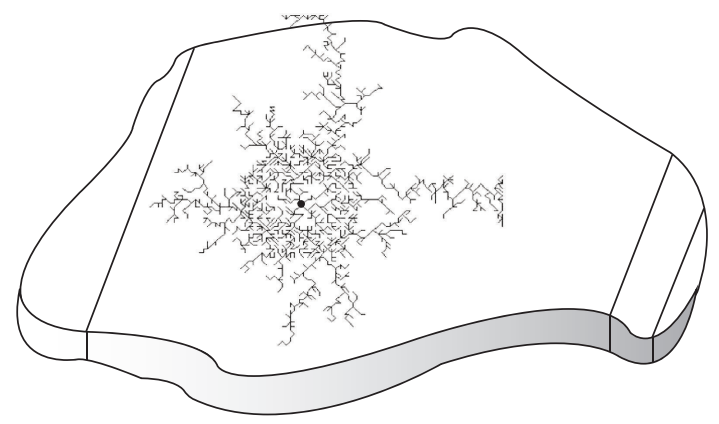

(b) $R=14$

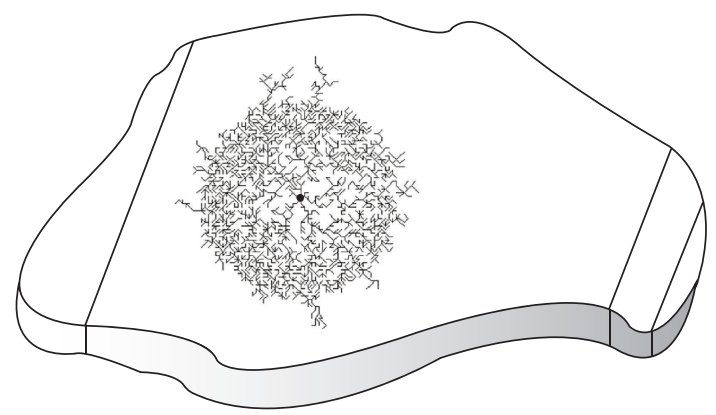

(d) $R=26$

FigURE 7: Fractal aggregation when $u=5 \times 10^{-5}\left(x^{2}+y^{2}\right), \xi=1 \times 10^{-7}$, and $p=3$.

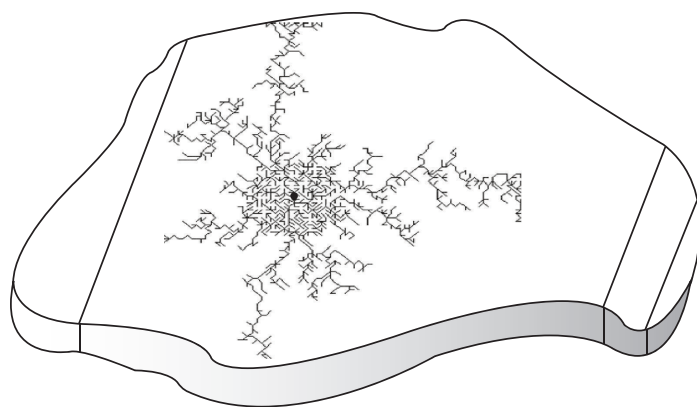

(a) $R=10$

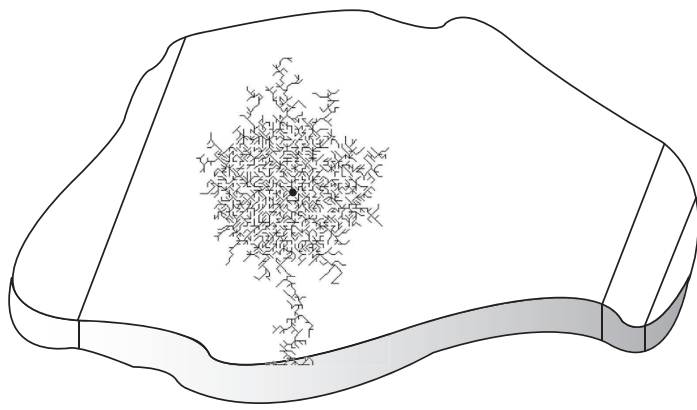

(c) $R=20$

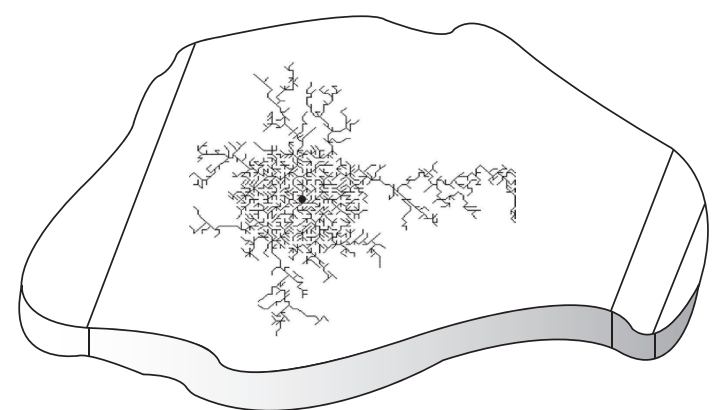

(b) $R=14$

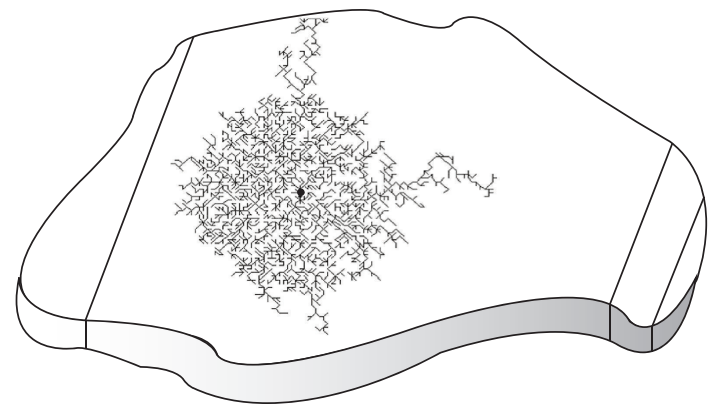

(d) $R=26$

FIGURE 8: Fractal aggregation when $u=0.01, \xi=1 \times 10^{-7}$, and $p=3$.

of engineering technology, methods for their prediction and control have great practical significance. In this paper, we investigate the continuous variation of thermal conductivity in the form of an exponential function. We also present a mathematical model for the environmental disturbance of thermal fractal growth and analyze the quantitative relationship between the growth probability and the disturbance term. Assuming that the environmental disturbance term 
TABLE 2: $a(x, y)=b(x, y)=e^{0.02 x}$, fractal dimension of thermal diffusion under different environmental disturbances.

\begin{tabular}{lcccc}
\hline Figure number & $\xi$ & Parameter & Fractal dimension \\
& $\xi$ & $u$ & $R$ & \\
\hline Figure 4(c) & 0 & 0 & 0 & 1.4456 \\
Figure 7(a) & $10^{-7}$ & $5 * 10^{-5}\left(x^{2}+y^{2}\right)$ & 10 & 1.1569 \\
Figure 7(b) & $10^{-7}$ & $5 * 10^{-5}\left(x^{2}+y^{2}\right)$ & 14 & 1.6921 \\
Figure 7(c) & $10^{-7}$ & $5 * 10^{-5}\left(x^{2}+y^{2}\right)$ & 20 & 1.795 \\
Figure 7(d) & $10^{-7}$ & $5 * 10^{-5}\left(x^{2}+y^{2}\right)$ & 26 & 1.9432 \\
Figure 8(a) & $10^{-7}$ & $10^{-2}$ & 10 & 1.4995 \\
Figure 8(b) & $10^{-7}$ & $10^{-2}$ & 14 & 1.5276 \\
Figure 8(c) & $10^{-7}$ & $10^{-2}$ & 20 & 1.653 \\
Figure 8(d) & $10^{-7}$ & $10^{-2}$ & 26 & 1.7866 \\
\hline
\end{tabular}

consists of a forced item with a polynomial form and a source item with a circular designated area, we use Matlab simulation to measure the control effectiveness of thermal fractal diffusion. This enables us to select appropriate control areas and control parameters for the variable coefficients of thermal diffusion. Our findings offer insight into the physical mechanisms of fractal growth in thin plates consisting of homogeneous layers, with applications in fields such as materials technology, medical science, and physics.

\section{Conflict of Interests}

The authors declare that there is no conflict of interests regarding the publication of this paper.

\section{Acknowledgments}

The project is supported by the National Natural Science Foundation of China (Grant nos. 61273088, 61473173, and 11201264 ), and also by Weihai Science and Technology Bureau (Grant no. 2012DXGJ22).

\section{References}

[1] X. Sun and Z. Q. Wu, Fractal Theory and Its Application, Press of University of Science and Technology of China, He'fei, China, 2003.

[2] X. Y. Wang and Y. Y. Sun, "The general quaternionic M-J sets on the mapping $\% \leftarrow \varkappa^{\alpha}+c(\alpha \in \mathrm{N})$," Computers and Mathematics with Applications, vol. 53, no. 11, pp. 1718-1732, 2007.

[3] X. Wang and T. Jin, "Hyperdimensional generalized M-J sets in hypercomplex number space," Nonlinear Dynamics, vol. 73, no. 1-2, pp. 843-852, 2013.

[4] X. Wang, Z. Wang, Y. Lang, and Z. Zhang, "Noise perturbed generalized Mandelbrot sets," Journal of Mathematical Analysis and Applications, vol. 347, no. 1, pp. 179-187, 2008.

[5] J. Sun and S. T. Liu, "A new identification control for generalized Julia sets," Chinese Physics B, vol. 22, no. 5, Article ID 050505, 2013.

[6] W. Xingyuan, J. Ruihong, and Z. Zhenfeng, "The generalized Mandelbrot set perturbed by composing noise of additive and multiplicative," Applied Mathematics and Computation, vol. 210, no. 1, pp. 107-118, 2009.
[7] X. Y. Wang and L. N. Gu, "Research fractal structures of generalized M-J sets using three algorithms," Fractals, vol. 16, no. 1, p. 79, 2008.

[8] X. Wang and Q. Liang, "Reverse bifurcation and fractal of the compound logistic map," Communications in Nonlinear Science and Numerical Simulation, vol. 13, no. 5, pp. 913-927, 2008.

[9] T. A. Witten and L. M. Sander, "Diffusion-limited aggregation," Physical Review B, vol. 27, no. 9, pp. 5686-5697, 1983.

[10] S.-H. Shiny, R. Hyeong, and C.-H. Kimx, "Hybrid simulation of Miscible mixing with viscous fingering," Computer Graphics Forum, vol. 29, no. 2, pp. 675-683, 2010.

[11] Y. Liu, Z. Liu, L. Huang, and J. Sun, "Fractal model for simulation of frost formation and growth," Science China Technological Sciences, vol. 53, no. 3, pp. 807-812, 2010.

[12] S. L. Shen, Analysis and Control of Diffusion Limited Aggregation Growth, Shandong University, Jinan, China, 2008.

[13] L. Zhang and S. T. Liu, "Control of thermal diffusion fractal growth of thin plate under environmental disturbance," Acta Physica Sinica, vol. 59, no. 11, pp. 7708-7712, 2010.

[14] X.-Y. Wang and W.-J. Song, "The generalized M-J sets for bicomplex numbers," Nonlinear Dynamics, vol. 72, no. 1-2, pp. 17-26, 2013.

[15] J. S. Andrade Jr., E. A. A. Henrique, M. P. Almeida, and M. H. A. S. Costa, "Heat transport through rough channels," Physica A: Statistical Mechanics and Its Applications, vol. 339, no. 3-4, pp. 296-310, 2004

[16] X. Y. Wang, W. Liu, and X. J. Yu, "Research on Brownian movement based on generalized Mandelbrot-Julia sets from a class complex mapping system," Modern Physics Letters B, vol. 21, no. 20, pp. 1321-1341, 2007.

[17] X.-y. Wang, P.-j. Chang, and N.-n. Gu, "Additive perturbed generalized Mandelbrot-Julia sets," Applied Mathematics and Computation, vol. 189, no. 1, pp. 754-765, 2007.

[18] X. Wang and P. Chang, "Research on fractal structure of generalized M-J sets utilized Lyapunov exponents and periodic scanning techniques," Applied Mathematics and Computation, vol. 175, no. 2, pp. 1007-1025, 2006.

[19] R. T. Li, Heat Transfer Theory and Method, Tianjin University Press, Tianjin, China, 1992.

[20] H. Liu, "The differential equations of heat transfer for some cases of variable thermal conductivities," Applied Mathematics and Mechanics, vol. 2, no. 4, pp. 379-385, 1981.

[21] H. C. Liu, "The differential equations of heat transfer for some cases of variable thermal conductivities," Applied Mathematics and Mechanics, vol. 2, no. 4, pp. 379-385, 1981.

[22] H. J. Zhang, Heat Conduction, Higher Education Press, Beijing, China, 1992.

[23] J. P. Lu and Z. Guan, Numerical Solution to Partial Differential Equations, Tsinghua University Press, Beijing, China, 2003.

[24] W. Qiao, S.-T. Liu, and J. Sun, "Control of thermal fractal diffusion of space body," Control Theory and Applications, vol. 31, no. 3, pp. 361-365, 2014.

[25] L. Pietronero and H. J. Wiesmann, "Stochastic model for dielectric breakdown," Journal of Statistical Physics, vol. 36, no. 5-6, pp. 909-916, 1984.

[26] L. Wang, Shop Scheduling with Genetic Algorithms, Tsinghua University Press, Beijing, China, 2003.

[27] B. K. Jiadeke, An Introduction to Uniform Polynomial Apporximation of Function, Beijing University Press, Beijing, China, 1989. 
[28] Z. C. Xue, S. Z. Yu, and J. Y. Yuan, Advanced Mathematics, Tsinghua University Press, Beijing, China, 2008.

[29] K. Falconer, Fractal Geometry, John Wiley \& Sons, New York, NY, USA, 2003. 


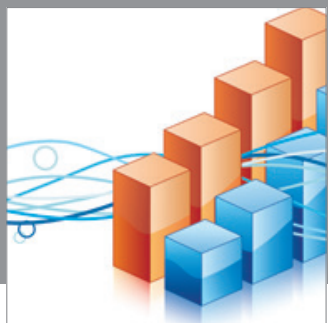

Advances in

Operations Research

mansans

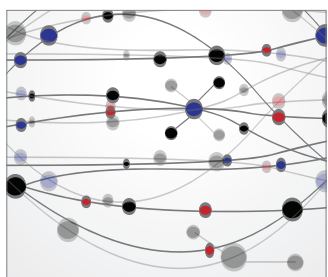

The Scientific World Journal
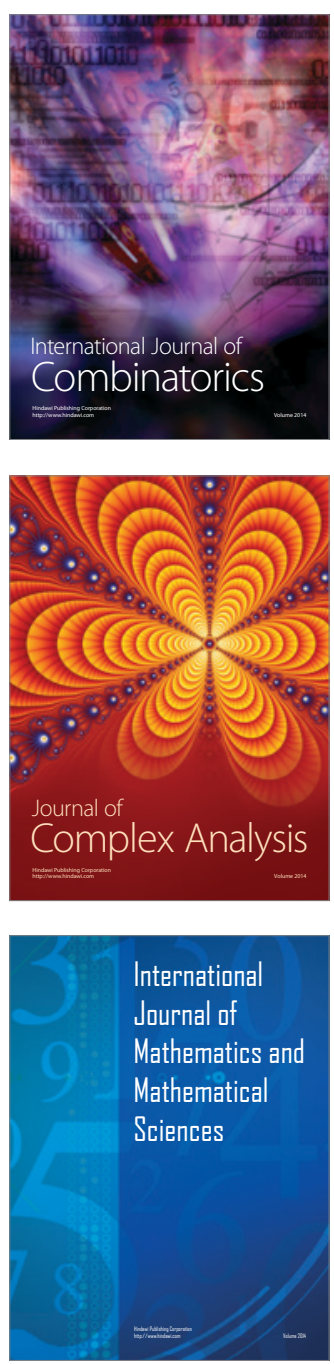
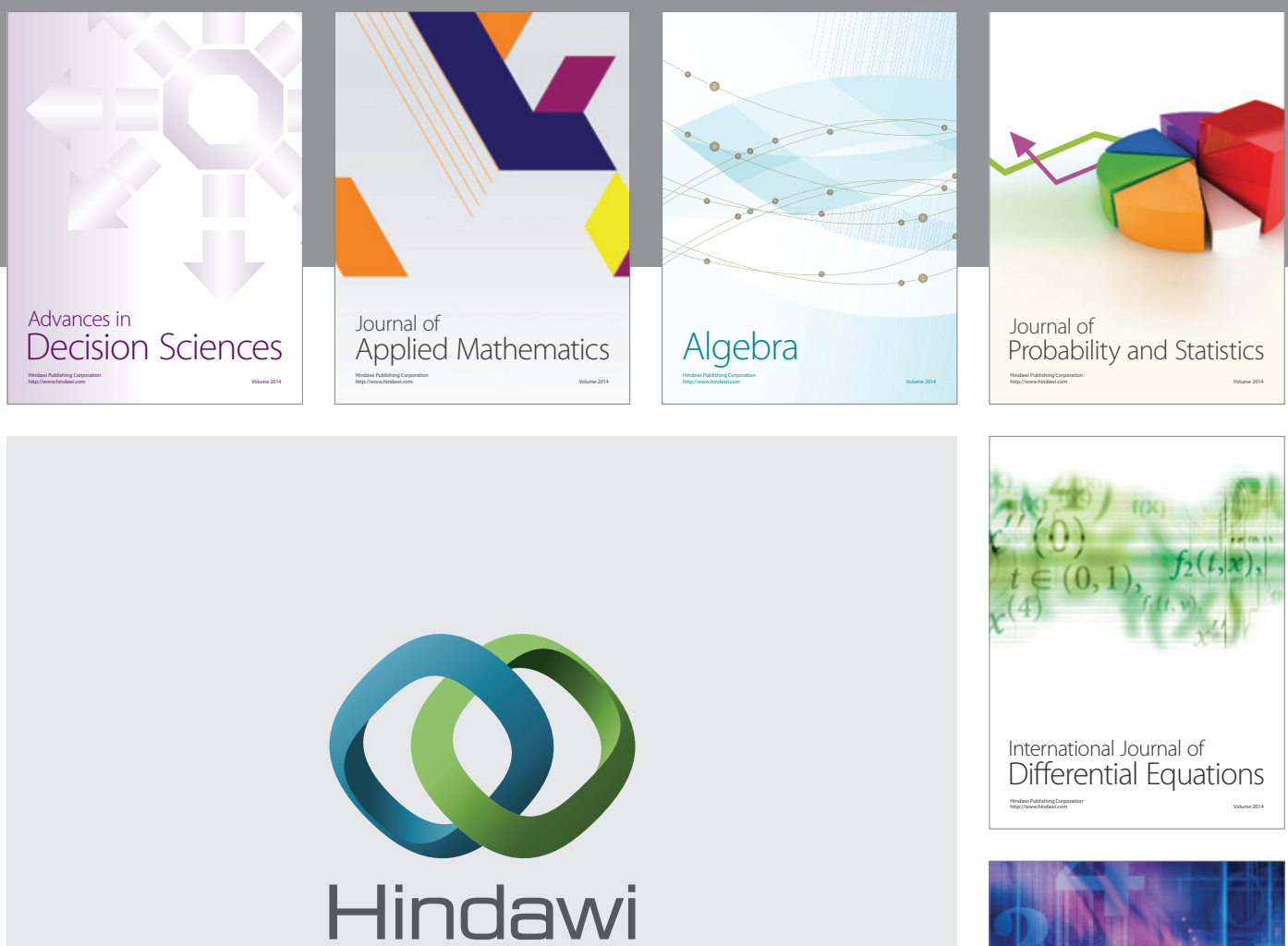

Submit your manuscripts at http://www.hindawi.com
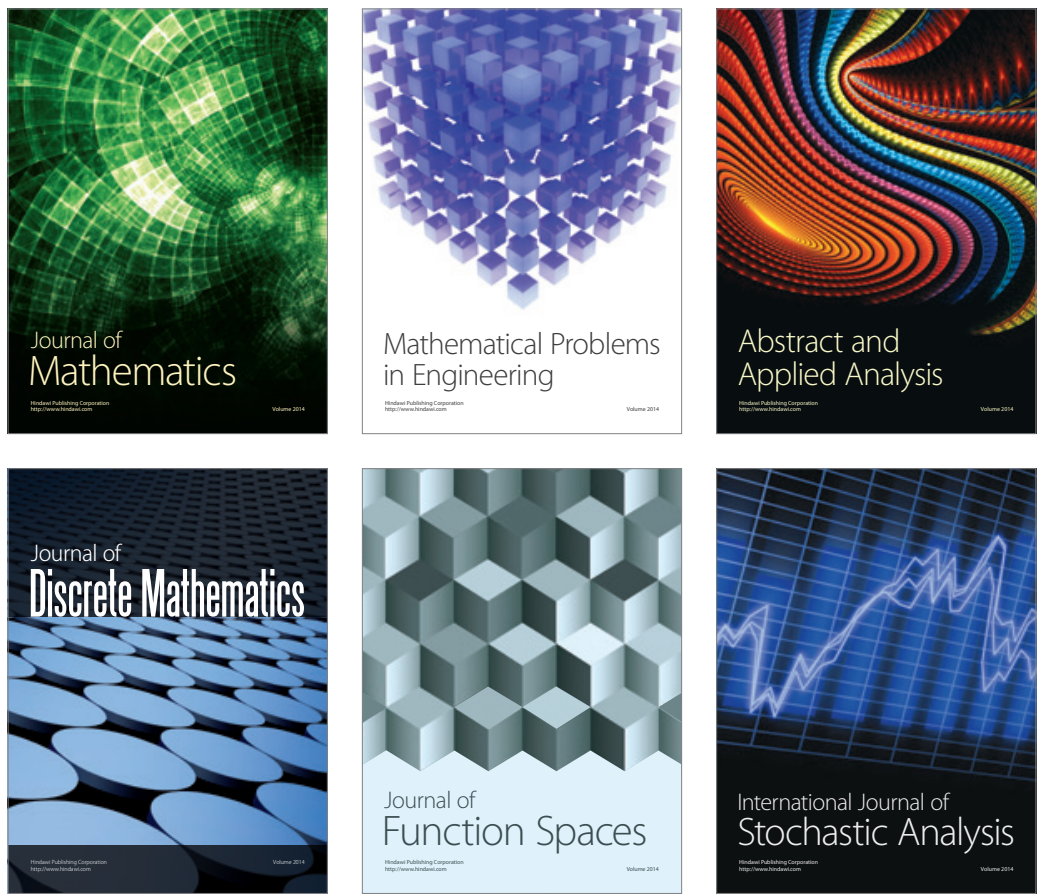

Journal of

Function Spaces

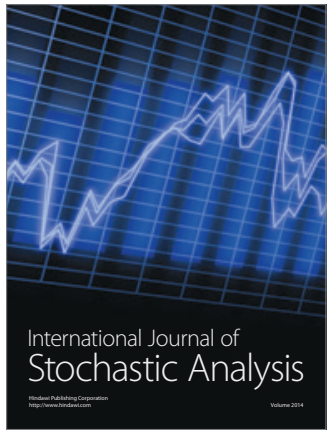

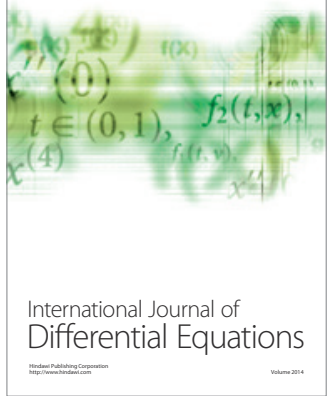
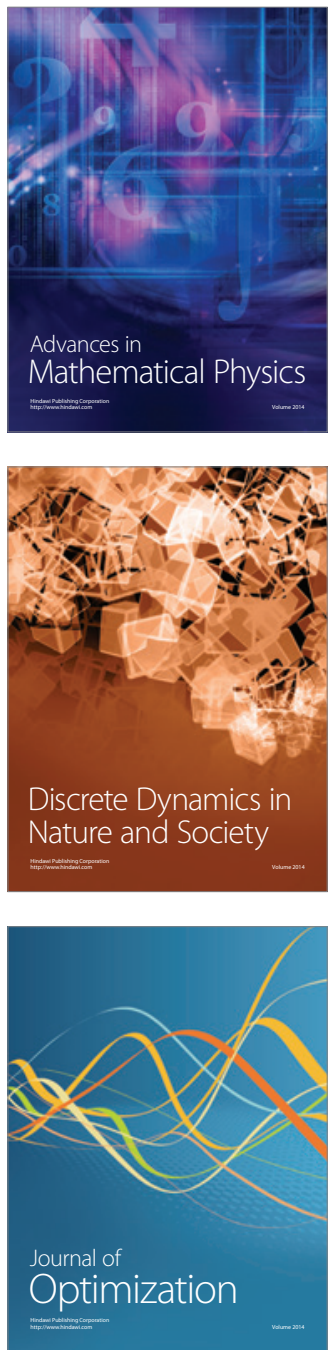